\title{
A LANGUAGE-BY-RADIO MEDIA PROGRAM
}

\author{
by Edmun B. Richmond, Georgia Institute of Technology
}

Since 1976, two-way radio communications have been used in experimental course designs in the foreign language curriculum to implement classroom exercises and to bring American students into immediate personal contact with native speakers of the target language. These courses were born out of the need to provide the student with more authentic language and cultural exposure than is found in the foreign language class itself. Research done in the field of foreign language education pointed toward the need to provide real-world language usage and cultural awareness on an individual and personal level, not simply pattern practice and contrived classroom conversations.

An innovative approach to adding these dimensions to the foreign language curriculum was developed and implemented through the use of two-way amateur radio contacts. This article will not attempt to describe these experimental courses in detail, but will give an overview of the work done in this field.' It will briefly describe (1) the original pilot course in German; (2) a replication of the pilot course in Spanish; (3) a curriculum-based German conversation course involving students at two universities, all of which utilized amateur radio as the means of communication; and (4) a language activity utilizing portable CB radio equipment.

\section{The Pilot Course}

In the spring quarter of 1976 , a pilot course was initiated at Georgia Tech for students enrolled in the third quarter of elementary German to listen and speak to native German speakers, who were radio amateurs. Since the study had no status as an actual course, no credit could be offered; as a result, attendance was voluntary.

The class met each Friday, from 12 noon to 1 p.m., at the Tech campus amateur club station. The first meeting was conducted with six students. During the session, the procedure for making contacts was explained. Since the students' command of German was still basic, it

NALLD Journal 
was recommended that they talk in simple utterances. In addition, it was explained to the German-speaking amateurs that they would have to speak clearly and keep their utterances simple in structure for optimum understanding.

The thrill of speaking to someone overseas, and the fact that the German operators called each student by first name (a common practice among hams), had an exhilarating effect on the students. They acted as the course's publicity agents, and within two weeks, the enrollment jumped to eight, and to eleven shortly after. This final enrollment held throughout the remainder of the quarter. Even with this number, each student had an opportunity to speak several times during the hour.

Conversational subjects ranged from simple introductions, and weather reports to descriptions of occupations, family make-up, student areas of study, and descriptions of surrounding geographical features. Most of the "conversation" emanated from the German operator, who gave willingly to this experiment. It was apparent that those operators would use some vocabulary with which the students were not familiar. Therefore, each student was asked to keep a list of unfamiliar words or phrases which were discussed by teacher and class during the last ten minutes of each hour.

One contact with the Technical University in Darmstadt was particularly interesting to the Tech students, since many of them were majoring in electrical engineering. This contact precipitated their asking for a German technical vocabulary of electronic terms, which they actually used in subsequent contacts.

Although no causal relationship was established, those students who participated in the pilot course did seem to generate a higher interest in the German language and culture, and did seem to demonstrate higher classroom motivation than students who did not participate in the course. These positive attitudes, plus the student critique at the end of the quarter, encouraged further development of such language-by-radio courses.

\section{The Spanish Course}

A similar course, this time offering one hour of credit, was promoted for students of Spanish. In the fall quarter of 1977, fifteen advanced level students registered for "Conversational Spanish via Amateur Radio," which met one hour per week. All fifteen students had completed at least two years of study in Spanish, and were enrolled in either third or fourth year courses in the department. They had already completed a course in Peninsular and Latin American history and culture, the department's standard second year course. This, along with a more advanced command of spoken Spanish, allowed radio contacts which went beyond the basic information exchanged in the German pilot study. 
Throughout the quarter, contacts were made with nine Latin American countries. Although students were encouraged to speak with the operators, many chose to listen for the first few weeks, until they became familiar with the various dialectical differences found in New World Spanish. As the students gained confidence, they ventured fourth to speak to the amateur at the other end of he radio circuit.

As in the German pilot course, all of the Spanish-speaking amateurs indicated a great interest in our utilizing amateur radio to gain cultural insights and linguistic competence. Each was extremely cooperative in describing in great detail his country's geography, natural resources, history, as well as personal descriptions of daily life, occupations, and hobbies.

Much cultural information was also obtained from these contacts. A round-table, multi-contact session developed during one class period. A Paraguayan amateur told of her country's national dances and indigenous musical instruments. While this contact was in progress, a station in Panama joined the conversation to describe the dances of his country. Within a few minutes, a station in Guatemala City joined in, after having listened to our conversation for about ten minutes, and contributed his country's musical heritage to the conversation. The students had an opportunity to hear three different dialectical variations of New World Spanish at the same time, and were further treated to the nationalistic pride exhibited by each of the three amateurs.

The students were amazed to discover that what they had read in their classes about the individual countries, was being verified by the amateur! The students had already been exposed to basic information, and had discussed it in class. It was material with which they were familiar, and which was reinforced through the radio contacts. In addition, new cultural information, which was not found in their texts, was received.

One interesting feature of the class was the students' discovery of the variety of dialects in New World Spanish. This prompted them to inquire into these regionalisms, and a general outline of Spanish dialectology was discussed over several class periods. As a direct result of this interest, the department will offer a three-hour course in Spanish dialectology next fall quarter.

A critique of the course was conducted at the end of the quarter. Again, the students considered their time to have been well spent. All agreed that their understanding of spoken Spanish and cultural awareness, as well as their speaking ability had improved. All wanted a continuation of the course, one which would meet two hours in succession, instead of the one hour. Some wanted to meet three times a week as well. 
The Georgia Tech-University of Louisville Experiment

During the same quarter in which the Spanish language-by-radio course was offered, a full-fledged curriculum-based conversational course in German was conducted between Georgia Tech and the University of Louisville. This course, which met once a week, focused on the conversational skills of advanced level students who were enrolled in German courses at their respective universities. Seven Georgia Tech students received one hour of credit for the course. The University of Louisville students participated on a voluntary basis and received no credit.

The course was pedagogically developed with distinctive conversational subjects for each week. These subject areas were geared to develop personal rapport between participants at both universities. Each student could share his own, personal background and feelings on the particular subject of the week. The subjects discussed were: Week One, student introductions, which included hobbies, interests, home-towns, areas of study, etc; Week Two, discussion of favorite types of transportation, i.e., cars, motorcycles, and bicycles; Week Three, a more detailed discussion of hobbies, favorite sports, and major interests; Week Four, a picture identification game based on "Twenty Questions;" Week Five, discussion of an article from a German weekly magazine; Week Six, a geography identification game; Week Seven, future life plans; Week Eight, a situation entitled, "If I had a millioin dollars . . .;" Week Nine, an oral final examination.

During the course, the role of each instructor was limited to keeping continuity so that conversations remained lively and active. In addition, they assisted in explaining any unfamiliar words or phrases to their students. However, an interesting phenomenon developed as the course continued. Instead of asking the instructor for his information, the students began helping each other, so that instructor involvement became less and less important. Each school's students asked the counterpart students personally-related questions, so that the course developed the true flavor of real conversation.

Constant pedagogical feedback was maintained throughout the quarter. Since this was an original and innovative approach to language teaching, it was necessary that both instructors remain in constant communication, not only in the development of he course, but also to provide feedback during the course. This was accomplished through the taping of each hour's class on cassette and maintaining telephone contacts after each week's class for a critique and to review and finalize the material for the coming week.

The final examination was based on the same format as the class. Each student was asked five questions. These questions were based on the material discussed during the class. Three questions were general in nature, and two were personally tailored to each student's background and interests. A list of thirty general questions were prepared by the instruc- 
tors, and were randomly chosen for each student. Both instructors graded each student according to a pre-arranged scale, and later compared and averaged their scores to obtain the final grade.

At the end of the quarter, a critique session was held on the air in English, which was also recorded. All participants agreed that the course was a great success and wanted to see its continuation. To this end, the course has been offered during each subsequent quarter with ascending enrollments.

\section{The Language Treasure Hunt}

Another means of utilizing radio communications to further foreign language practice uses hand-held citizen's band (CB) units in a variation of the treasure hunt party game. This allows language-by-radio activity to be initiated from the classroom itself, and can be used in addition to, or instead of, the contacts by amateur radio.

The treasure hunt allows group activity and generates team spirit. It involves finding a coded or scrambled message in the target language, which is hidden by the teacher. Each team has two CB handi-talkie units, one for a base team, which remains with the teacher, and one for a field team, which does the searching. The base team knows the location of the hidden message, and must relay instructions how to get there, in the target language, to the field team. Once the message is found, it must be decoded by the field team and transmitted back to the base team. When both base team and field team are certain they have decoded the message properly, the base team writes the message on paper and hands it to the teacher, who confirms or rejects the message.

This activity integrates the "four skills" of language learning, i.e., listening, speaking, reading, and writing. The game can be utilized as a competition between teams, different classes, across languages (a French class can challenge a German class), or across schools. It is an ideal activity for language clubs or "language fair" contests.

The actual license for this activity is obtained by the language department itself, free of charge from the Federal Communications Commission. Any FCC field office can supply license application form 505. It is filled out and mailed to the FCC. When received, the license allows up to 25 individual $C B$ units to be used under the same callsign. The hand-held units are inexpensive, easy to obtain, and easy to use.

Implications for Further Educational Use

The radio communications described in this article are ideal for foreign language study and practice. They generate not only the personal communicative aspect, but also allow students to gather much cultural information not readily found in textbooks. It would follow that other types of classes, such as history, geography, social studies, and the like could make use of a radio program, with contacts in English, to enhance 
and support the classroom curriculum. If a unit on Africa is being studied, contacts from that continent would bring the information closer to the students, and would provide a more meaningful and personal contact for them. That area would no longer be an impersonal entity whose contact from a book is far removed from the real place.

This radio communication can, therefore, do much to further personal international contact. Most high schools and colleges have a ham club station in operation which can be used for this purpose. From the success of these experimental courses, it appears that such a language-by-radio media program has a valid place in educational planning.

\section{Notes}

'For a complete description of these language-by-radio courses, see Edmun B. Richmond and Dale V. Lally, Jr., German via the Airwaves: A Foreign Language Experiment that Worked!, Foreign Language Annals, 1978, 6, 687-691; Ećmun B. Richmond, Amateur Radio as an Aid to Foreign Language Learning, Foreign Language Annals, 1978, 3, 259-263; Edmun B. Richmond, Amateur Radio in the Foreign Language Curriculum: Media Usage on an International Scale, International Journal of Instructional Media, 1977-78, 5(1)( 45-50. A description of the Language Treasure Hunt utilizing $C B$ radio is forthcoming in Foreign Language Annals. 